\title{
Gap junctions remain open during cytochrome $c$-induced cell death: relationship of conductance to 'bystander' cell killing
}

\author{
K Cusato $^{\star, 1,3,5}$, H Ripps $^{2,3,4}$, J Zakevicius ${ }^{2,3}$ and DC Spray ${ }^{1}$ \\ ${ }^{1}$ Department of Neuroscience, Albert Einstein College of Medicine, Bronx, \\ NY 10461, USA \\ 2 Department of Ophthalmology and Visual Sciences, University of Illinois \\ College of Medicine, Chicago, IL 60612, USA \\ ${ }^{3}$ Marine Biological Laboratory, Woods Hole, MA 12543, USA \\ ${ }^{4}$ Department of Anatomy and Cell Biology, University of Illinois College of \\ Medicine, Chicago, IL 60612, USA \\ 5 Present address: Department of Ophthalmology, UCSF, San Francisco, \\ CA 94143, USA \\ * Corresponding author: $\mathrm{K}$ Cusato, Department of Ophthalmology, University of \\ California, San Francisco, 10 Koret Way, Box 0730, San Francisco, CA 94143, \\ USA. Tel: + 415-476-6097; Fax: + 415-476-6289; \\ E-mail cusatok@vision.ucsf.edu
}

Received 12.10.05; revised 06.12.05; accepted 04.1.06; published online 17.2.06 Edited by RA Knight

\begin{abstract}
Previous reports have shown that gap junctions relay cell death in many cell types. However, changes in electrical coupling and their dynamics during cell death are poorly understood. We performed comprehensive studies of electrical coupling following induction of cell death by single-cell cytochrome $c$ (cyC) injection in paired Xenopus oocytes. Cell death was rapidly induced by cyC in injected cells, and cell death was also observed in uninjected bystander cells electrically coupled to the cyC-injected oocytes. Gap junction currents either remained at pre-cyC injection levels or increased dramatically as the injected cell died. Nonjunctional currents increased in injected cells immediately following cyC injection; nonjunctional currents increased slowly in uninjected bystander cells. Bystander cell death occurred only when junctional conductance was $\sim 6 \mu \mathrm{S}$. Both 1,2-bis(o-aminophenoxy)-ethane- $N, N,-N^{\prime}, N^{\prime}$-tetraacetic acid tetraacetoxy-methyl ester and Xestospongin $C$ inhibited bystander cell death in pairs that had reached the death conductance threshold, suggesting that $\mathrm{Ca}^{2+}$ and inositol 1,4,5 triphosphate are involved in the process.

Cell Death and Differentiation (2006) 13, 1707-1714. doi:10.1038/sj.cdd.4401876; published online 17 February 2006
\end{abstract}

Keywords: cell death; intercellular communication; gap junctions; bystander effect; Xenopus oocytes

Abbreviations: BAPTA-AM, 1,2-bis-(o-aminophenoxy)-ethane$N, N,-N^{\prime}, N^{\prime}$-tetraacetic acid tetraacetoxy-methyl ester; Cx, connexin; anti-Cx38, antisense oligonucleotides to $\mathrm{Cx38}$; cyC, cytochrome $c$; $G_{\mathrm{j}}$, gap junctional conductance; $G_{\mathrm{nj}}$, nonjunctional conductance; $\mathrm{G}_{\text {total }}$, total conductance; $\mathrm{IP}_{3}$, inositol 1,4,5 triphosphate; MB, Modified Barth's Solution

\section{Introduction}

Throughout the nervous system, many classes of neurons undergo apoptosis, or programmed cell death. Numerous mechanisms trigger this process both in the course of normal development and in a broad range of pathological conditions. ${ }^{1-3}$ We previously reported that gap junctional communication performs a novel role in the intercellular propagation of naturally occurring developmental cell death ${ }^{4}$ and there is strong evidence that gap junctions mediate the spread of cell death in experimentally induced apoptosis. ${ }^{5,6}$ These studies represent examples of an unusual, but not uncommon phenomenon known as the 'bystander effect'. The term was introduced by Freeman et $\mathrm{al}^{7}$ to describe the findings observed during gene therapy for primary brain tumors. In that study, the thymidine kinase gene from herpes simplex virus was used in combination with the nucleoside analog, gancylovir, thereby enzymatically converting the nontoxic prodrug into a lethal compound that inhibited the growth of gene-transfected cells. Owing to the low efficacy of viral vectors, only a fraction of the tumor mass contained the viral gene, but it had induced cell death throughout the tumor. In brief, the therapeutically destructive effect induced in the successfully transfected cells had spread to 'bystanders', that is, to cells throughout the tumor that otherwise would be expected to survive and multiply. There is strong evidence, both from in vitro and in vivo studies, that the bystander effect is mediated via the gap junctions formed by the connexins expressed in tumor cells. ${ }^{8-12}$

Gap junction channels are intercellular communication pathways formed by a multigene family of homologous proteins known as connexins. Six connexin polypeptides oligomerize to form a membrane hemichannel or connexon, which docks with a connexon from an adjacent cell to create an aqueous pore $(d \approx 1.6 \mathrm{~nm})$ that bridges the $\sim 3 \mathrm{~nm}$ intercellular 'gap'. Once formed, the gap junctional channel allows the cell-to-cell diffusion of ions, second-messenger molecules, metabolites, and small peptides having a molecular mass $\leq 1 \mathrm{kDa} .{ }^{13-15}$ Clearly, proapoptotic factors such as $\mathrm{Ca}^{2+}$ and reactive oxygen species can readily pass through gap junction channels to exert their effects on neighboring cells, whereas the molecular size of proteins directly involved in the apoptotic pathway, for example, cyC, Apaf-1, and the caspases, preclude their passage through these intercellular channels.

Intrinsic changes in both the intracellular and extracellular environment act to open or close gap junction channels, and thus modulate overall junctional permeability and ionic conductance. This dynamic aspect may affect the vulnerability of neighboring cells to toxic agents and be of paramount importance in determining the rate and extent of the spread of cell death. Results obtained from the study of glioma cell lines undergoing bystander killing indicate that cell death is 
accompanied by a deregulation of intracellular calcium, ${ }^{16}$ and gap junction permeant dyes have been used to show that dye spread continues between dying cells and healthy neighbors in epithelial cell lines, ${ }^{17}$ as well as in cerebral cortex following ischemia. ${ }^{16}$ Although these studies suggest that gap junctional communication persists during cellular injury, relatively little is known of how gap junctions react to the dramatic intracellular changes that occur during cell death. Thus, it is unclear as to whether there are significant changes in junctional conductance during the process of cell death and which molecules and signaling pathways are potentially involved in communicating the death signal to bystanders.

The results of the present study demonstrate that electrical coupling between pairs of Xenopus laevis oocytes was retained following induction of cell death in one cell, that it persisted until the cell had degenerated, and that in many cases the intercellular conductance increased. Moreover, death of the bystander cell also occurred, which was inhibited by 1,2 -bis- (o-aminophenoxy)-ethane- $N, N,-N^{\prime}, N^{\prime}$-tetraacetic acid tetraacetoxy-methyl ester (BAPTA-AM), a cell permeant $\mathrm{Ca}^{2+}$ chelator, and Xestospongin $\mathrm{C}$, an inositol 1,4,5 triphosphate $\left(\mathrm{IP}_{3}\right)$ receptor inhibitor, suggesting that $\mathrm{Ca}^{2+}$ is a mediator of bystander cell death.

\section{Results}

\section{Cytochrome $c$ injection induces both primary and bystander cell death}

In order to study bystander killing, one cell of an electrically coupled pair of oocytes from $X$. laevis was induced to die by injection of cytochrome $c(\mathrm{cyC})$. cyC-induced cell death has been demonstrated in multiple cell types, ${ }^{18,19}$ and the technique has proven useful in studies of bystander cell death. ${ }^{4-6}$ In the Xenopus oocyte, intracellular injection of cyC leads to the rapid activation of caspases, changes in pigmentation, and a progressive loss of resting membrane potential. ${ }^{20}$ In a series of control experiments in which oocytes were injected with the antisense nucleotide to $\mathrm{Cx38}$ and placed in contact at their vegetal poles, every oocyte injected with cyC died over the course of $\sim 1-2 \mathrm{~h}(n=9)$, but since gap junctions failed to form, the neighboring cell remained intact throughout the day. These experiments show not only that intercellular communication is required for bystander cell death, but also that in these circumstances, the extrusion of potentially toxic substances from the dying cell did not cause the death of its neighbor.

An example of cyC-induced cell death is illustrated by the paired cells in Figure 1, which shows the disruption of structural integrity (Figure $1 \mathrm{a}-\mathrm{d}$ ) and the progressive loss of membrane potential (Figure 1e) that occur both in injected cells and in the cells to which they are electrically coupled. Prior to the introduction of cyC, cells appeared healthy and uniformly pigmented despite removal of their follicular and vitelline membranes, and having been impaled by a microinjection pipette (cell at right in Figure 1a). Within 20-30 min after injection (Figure 1b), the injected cell showed a loss of pigmentation at the animal pole and began to swell. At $60 \mathrm{~min}$, the swelling had increased (Figure 1c), and after 90-120 min (Figure 1d), the membrane of the injected cell became
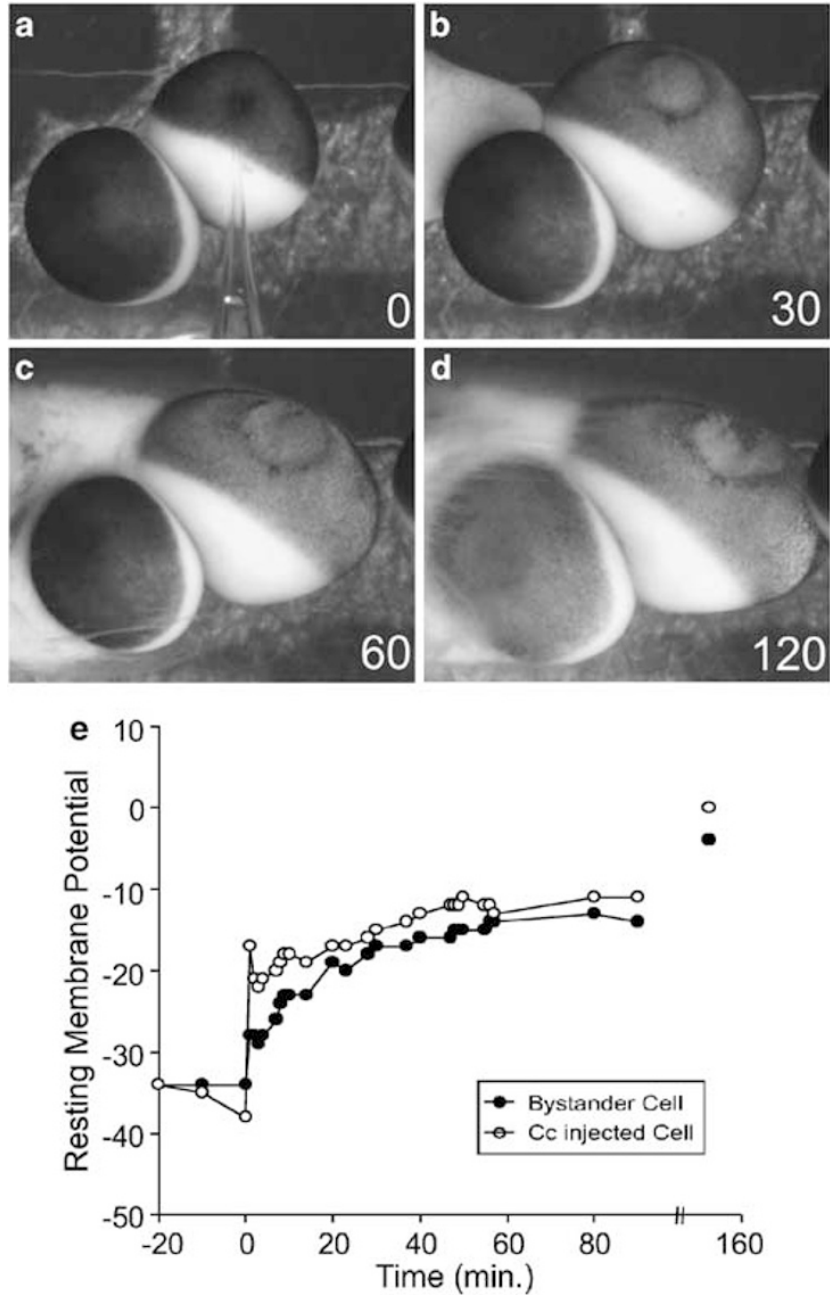

Figure 1 Cytochrome $c(\mathrm{cyC})$ injection causes primary and bystander cell death. (a-d) Time lapse photographs of electrically coupled oocytes following injection of cyC into the cell at the upper right. (a) At the time of injection, both cells have normal pigmentation and morphology. (b) At 30 min after introducing $\mathrm{cyC}$, the injected cell shows a loss of pigmentation at the animal pole and has begun to swell. (c) At 60 min post-cyC, the injected cell continues to swell and becomes somewhat distorted. (d) At 120 min post-cyC, the injected cell has lost structural integrity and the uninjected 'bystander'cell has begun to lose pigmentation and swell. (e) Time course of loss of membrane potential following cyC injection at $t=0$ into one cell of a pair. Both cells depolarize in response to the injection into one cell, but membrane potential decays slowly, reaching $\sim 0 \mathrm{mV}$ by $160 \mathrm{~min}$ post-cyC

disorganized and showed some out-pocketing; at the same time, pigmentary changes appeared in the coupled cell. The time course of cell death varied in cyC-injected cells, with some undergoing complete degeneration rapidly $(<1 \mathrm{~h})$, while others appeared relatively intact for as long as $2 \mathrm{~h}$. It is not clear what accounts for this variability, although differences in cell size and corresponding differences in cyC concentration may be a factor. Nevertheless, the rapid time course of cell death following direct microinjection of cyC is comparable to that observed in previous studies. For example, injection of only $10 \mu \mathrm{M}$ cyC in Xenopus oocytes produced cell death in more than $40 \%$ of the cells in less than $2 \mathrm{~h}^{20}$ Since we injected $14-28 \mu \mathrm{M}$ cyC, it is not surprising to find that cells were dying within 1-2 $\mathrm{h}$ postinjection. Moreover, 
a previous study showed that $20 \mu \mathrm{M}$ cyC induced apoptosis in a wide range of different cell types (e.g., clone 8 fibroblasts, IPC-81, Swiss 3T3 fibroblasts, NRK, HEK293, Y1, H295), and that apoptotic changes were seen in a large fraction of each cell line $1 \mathrm{~h}$ after injection. ${ }^{18}$ Rapid onset of cell death following cyC loading has also been observed in tissue. Cusato et al. ${ }^{4}$ reported that both caspase 3 cleavage and nuclear pyknosis occurred in the retinal explants within $1 \mathrm{~h}$ following cyC scrapeloading or single-cell microinjection. Clearly, our results are in line with these observations.

The changes in membrane potential that accompanied the apoptotic process were also variable, but typically the loss of potential occurred rapidly in the injected cell, whereas it began after a brief lag and progressed more gradually in the uninjected (bystander) cell (Figure 1e). However, it should be noted that not all cell pairs exhibited bystander cell death. Although cell death occurred in every cell of an electrically coupled pair that was injected with cyC $(n=15)$, only about $50 \%$ of the uninjected cells of the pairs displayed pigment loss and structural changes. As described below, this seeming inconsistency appears to result from variability in junctional conductance displayed by the various cell pairs during the time frame over which the membrane integrity of the injected cell was lost.

\section{Gap junctions remain open during cell death}

To examine intercellular communication during bystander cell killing, gap junction currents were recorded from paired oocytes using a dual-cell two-electrode voltage clamp paradigm (Figure 2a) before and after microinjection of cyC into one cell of the electrically coupled pair. An example of the current traces recorded from the two cells at selected time points before and after cyC injection (Figure $2 \mathrm{~b}$ ) shows that the gap junctional and total current (sum of junctional and nonjunctional currents) responses to hyperpolarizing voltage pulses increased in both cells over a 90 min time course. The gap junctional current increased to a maximum at $\sim 70 \mathrm{~min}$ postinjection, after which the junctional current decreased. The current necessary to hold the cyC-injected cell at $-40 \mathrm{mV}$ was $\sim 0.0064 \mu \mathrm{A}$ before cyC injection $(-10 \mathrm{~min})$, whereas postinjection, the holding current was $-0.391 \mu \mathrm{A}$ at $50 \mathrm{~min}$, and $-0.185 \mu \mathrm{A}$ at $90 \mathrm{~min}$. This is in contrast to the gap junctional current, which peaked at about $70 \mathrm{~min}$ and declined thereafter, indicating that the increase in junctional current was not simply a function of the cyC-injected cell becoming more 'leaky' (Figure 2b). This pattern of recovery of the holding current in the injected cell did not occur in all pairs; in many cases the holding current became larger until the cell could not be voltage clamped. The uninjected cell displayed a slow change in the holding current, starting at $-0.0025 \mu \mathrm{A}$ ( $-10 \mathrm{~min})$, then dropped slowly to $-0.034 \mu \mathrm{A}(50 \mathrm{~min})$ and finally to $-0.074 \mu \mathrm{A}$ (90 min), indicating that it too had become more 'leaky.'

The current recordings enabled us to calculate both the junctional $\left(G_{\mathrm{j}}=l_{\mathrm{j}} / V_{\mathrm{j}}\right)$ and nonjunctional $\left(G_{\mathrm{nj}}=\left(I_{\text {total }}-l_{\mathrm{j}}\right) / V_{\mathrm{j}}\right)$ conductances, where $G_{\mathrm{j}}$ is the gap junctional conductance and $G_{n j}$ the nonjunctional conductance. For the cell pair in Figure $2 \mathrm{~b}$, the cyC-injected cell showed large increases in nonjuctional conductance, but the corresponding changes
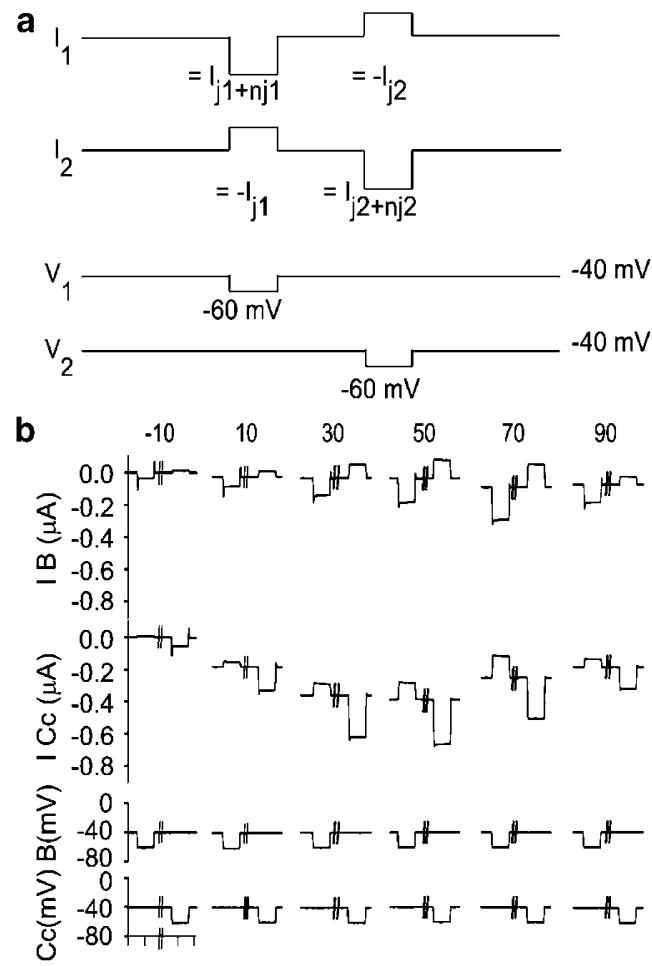

Figure 2 Gap junctional coupling measurements. (a) Protocol for dual cell twoelectrode voltage clamp studies. Cells were held at $-40 \mathrm{mV}$ and stepped to $-60 \mathrm{mV}$ for $1 \mathrm{~s}$. The downward deflection of the stepped cell is the total current $\left(G_{\text {total }}\right)$, which is the sum of the junctional current $\left(G_{\mathrm{j}}\right)$ and nonjunctional current $\left(G_{\mathrm{nj}}\right)$. The upward deflection in the follower cell is the current that has passed from one cell to the other via gap junctions $\left(G_{\mathrm{j}}\right)$. (b) Current recordings from a pair of cells in which one cell was injected with cyC at $t=0 \mathrm{~min}$. The $G_{\mathrm{j}}$ and $G_{\mathrm{nj}}$ in the cyC-injected cell (I cyC) increase as the cell dies. The bystander cell also undergoes an increase in $G_{j}$ equal to that of the cyC-injected cell (I B), but the increase in $G_{n j}$ is less pronounced. Bottom two traces show the timing of the voltage pulses to each cell, which were $1 \mathrm{~s}$ in duration and $10 \mathrm{~s}$ apart

in the uninjected cell were small by comparison (Figure 3a). Thus, the increase in gap junction current accounts for the majority of the increased total current in the uninjected cell in response to the voltage pulse (Figure $2 b$ ). This relationship was consistent whether the bystander cell died or not, and was also apparent following vehicle injections (not shown). It is likely that the nonjunctional conductance in the uninjected cell would have increased as the cell died, but we were unable to voltage clamp the cyC-injected cell beyond $90 \mathrm{~min}$, and therefore were unable to confirm this. Between recordings, the cells were not voltage clamped, which allowed us to follow the resting membrane potentials as cell death ensued. Figure 1e shows the membrane potential for the pair of cells described in Figures 2 and 3. Despite the fact that the nonjunctional conductances were much larger in the cyCinjected cell, the changes in membrane potential were similar in the injected and uninjected cells. Clearly, the cyC-injected cell was able to partially maintain its membrane potential despite its decreased input resistance.

Calculating the input resistance $\left(1 / G_{\text {total }}=1 /\left(G_{\mathrm{nj}}+G_{\mathrm{j}}\right)\right)$ (total conductance $\left(G_{\text {total }}\right)$ ) as a function of time (Figure $3 b$; $n=4$ ) showed that there was a loss of input resistance in both cells, with the minimum resistance of the injected cell reaching 

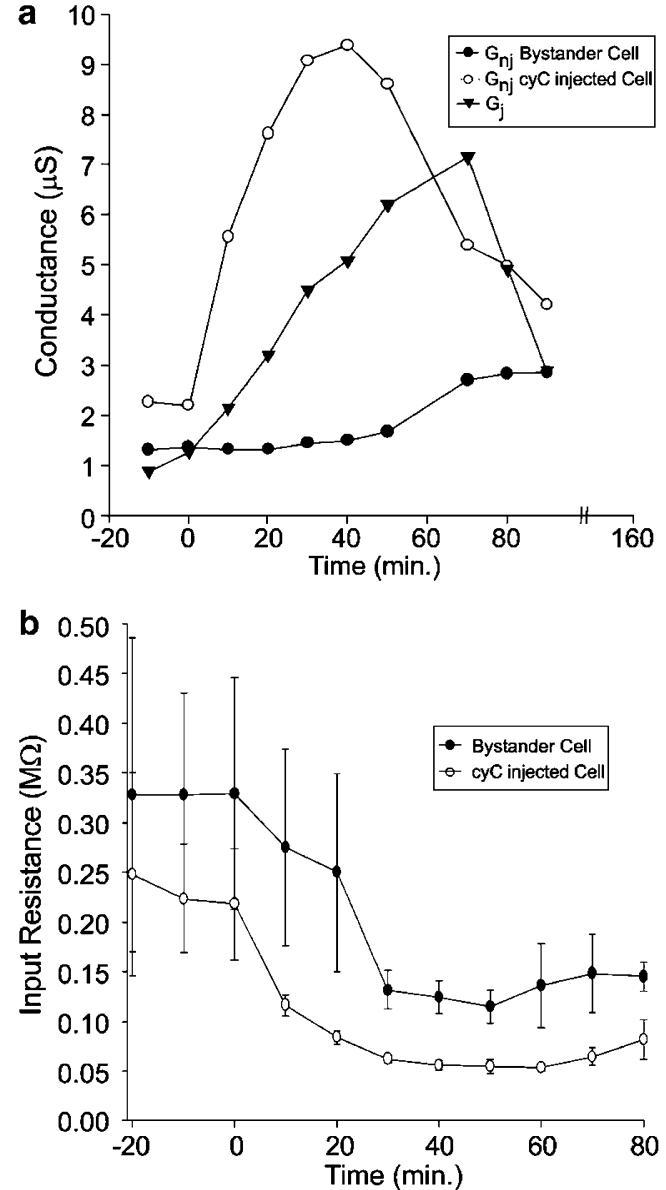

Figure 3 (a) Changes in $G_{n j}$ in a bystander and cyC-cell across time compared to the changes in $G$ between the cells, following cyC injection at $t=0 \mathrm{~min}$ (same pair of cells shown in Figure 2b). $G_{i}$ and $G_{n j}$ increase at different rates and peak at different times indicating that the increase in $G_{i}$ is not an artefact of the increase in $G_{n j}$. (b) Changes in input resistance of cyC-injected and bystander cells. Data represent the mean + I-S.E.M. calculated for each time point from four pairs of cells. Input resistance in the cyC-injected cell decreased following cyC injection as expected, reaching a minimum of $50 \mathrm{k} \Omega 30 \mathrm{~min}$ postinjection. There is surprisingly little variability in the cyC-injected cell, as evidenced by the error bars. Bystander cells also lost input resistance, regardless of whether the bystander cell survived or not, as this data includes both cases

$\sim 0.050 \mathrm{M} \Omega$. Despite this low resistance, it appears that the junction was still voltage clamped. The fact that the nonjunctional conductance peaked before the $G_{j}$ (Figure $3 a$ ) is a good indication that the junctional conductance cannot be an artefact of the increased nonjunctional conductance.

When we compared the responses of oocyte pairs $(n=15)$ to cyC injection by plotting the temporal changes in junctional conductance, it was apparent that the cells fell into three categories (Figure $4 \mathrm{a}-\mathrm{c}$ ). Some pairs showed large, rapid increases in gap junctional coupling, with the maximum conductance ranging between 9 and $14 \mu \mathrm{S}$ (Figure 4a), others had intermediate, slower increases with maxima between 5 and $7 \mu \mathrm{S}$ (Figure 4b), and some showed relatively small changes, with a maximum conductance of $<3 \mu \mathrm{S}$ (Figure $4 \mathrm{c}$ ). The changes seen in the last group were only slightly greater than those obtained for pairs of cells which had been previously injected with the antisense oligonucleotide to
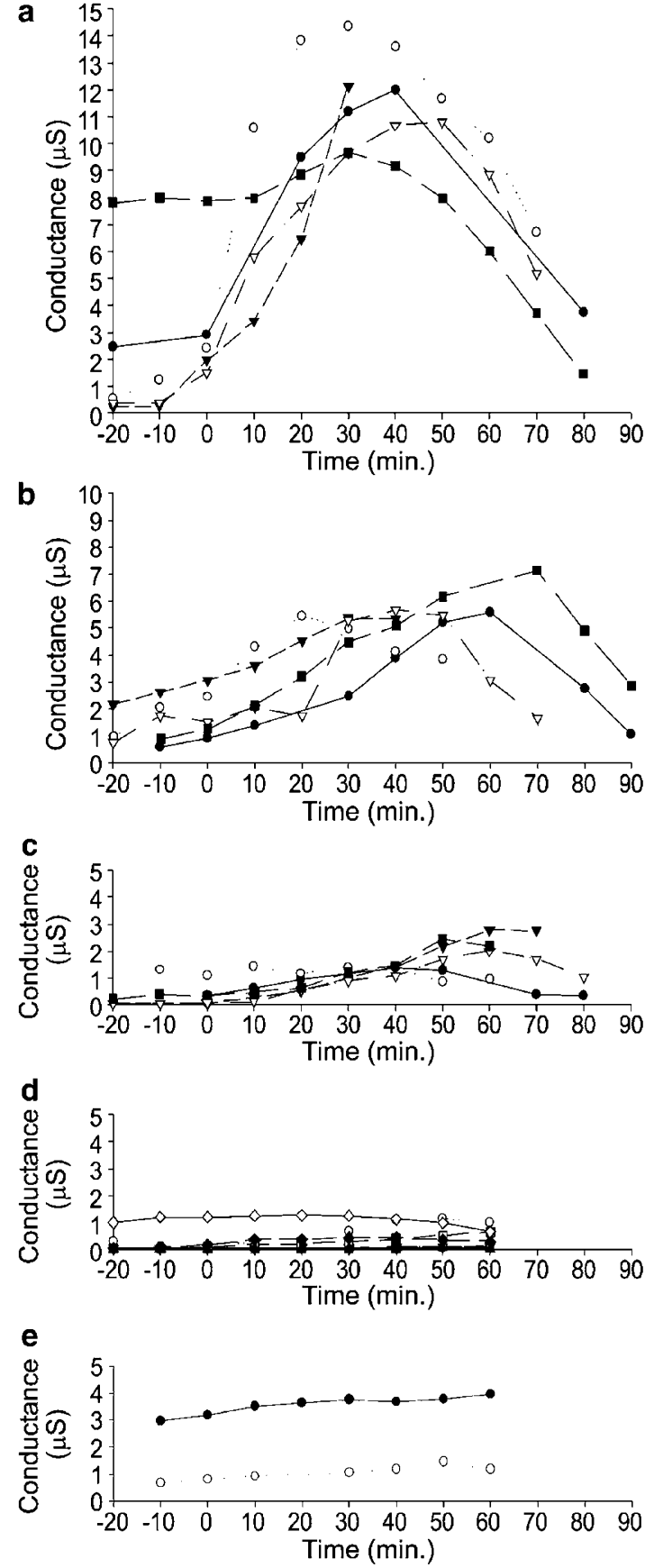

Figure 4 Changes in $G_{i}$ following cyC injection into one cell in each pair at $t=0$. Each line represents the data from a single pair of cells across time. Three populations of responses were observed. In some pairs, coupling increased dramatically $(\mathbf{a} ; n=5)$ and all bystander cells died. There were pairs that showed an intermediate response $(\mathbf{b} ; n=5)$ in which three of the bystander cells died. Some pairs responded with only a slight increase in coupling $(\mathbf{c} ; n=5)$; none of the bystander cells died. Injecting cells with antisense oligonucleotides to $\mathrm{Cx38}$ prior to manual pairing decreased $G_{i}(\mathbf{d} ; n=9)$, and none of the bystander cells died. Vehicle injections did not lead to increases in $G_{j}(e)$

Cx38 (Figure 4d), which effectively suppressed the formation of gap junctions. It should be noted that a loss of coupling from pre-cyC injection levels was never observed until the injected 
cell was losing membrane potential or lost structural integrity. Oocyte pairs always maintained or increased their junctional conductance during death of the injected cell. Some pairs exhibited small increases in current prior to cyC injection, but when this occurred, there was a further increase following cyC injection, and the rate of increase accelerated (Figure $4 \mathrm{a}$ and b). Following vehicle injection (intracellular solution lacking cyC), current increases were modest, with less than a twofold change in conductance (Figure 4e).

\section{Gap junctional conductance relates directly to bystander cell death}

By determining the maximum $G_{j}$ of each pair, and its relation to the survival or death of the uninjected cell, it became apparent that the level of conductance was an important predictor of the outcome (Figure 5). For pairs in which the maximum conductance was less than $3 \mu \mathrm{S}$, all uninjected (bystander) cells survived. In pairs that exhibited conductances in excess of $\sim 10 \mu \mathrm{S}$, the bystander cell always died. However, of the five pairs in which the junctional conductance reached an intermediate conductance level $(\sim 5-7 \mu \mathrm{S})$, three of the bystander cells died, and two survived. It appears that oocyte pairs must reach a threshold of junctional conductance $(\sim 6 \mu \mathrm{S})$ for evidence of bystander cell death. It should be noted that in all but one case, the pairs had less than threshold conductance prior to cyC injection, and in more than half of the cells studied, the conductance increase was sufficient to enable the cyC-injected cell to transmit a death signal to its coupled neighbor.

\section{$\mathrm{Ca}^{2+}$ plays a role in bystander cell killing}

To test whether elevation in cytoplasmic $\left[\mathrm{Ca}^{2+}\right]_{i}$ plays a role in bystander cell death, cells were incubated in the membrane permeant $\mathrm{Ca}^{2+}$ chelator, BAPTA-AM $(100 \mathrm{nM}$ or $10 \mu \mathrm{M})$, for 1h. An increase in $G_{j}$ was observed following BAPTA-AM treatment (Figure $6 a$ and $b$ ). Cells were then injected with cyC as in previous experiments and cell death was observed. Based on the findings shown in Figure 5, we considered $6 \mu \mathrm{S}$ as the conductance threshold for bystander cell death, and therefore, only pairs which reached this threshold before, during, or after BAPTA-AM incubation were considered in the analysis. Cell pairs that were not above the $6 \mu \mathrm{S}$ threshold at the time of cyC injection were followed through the course of

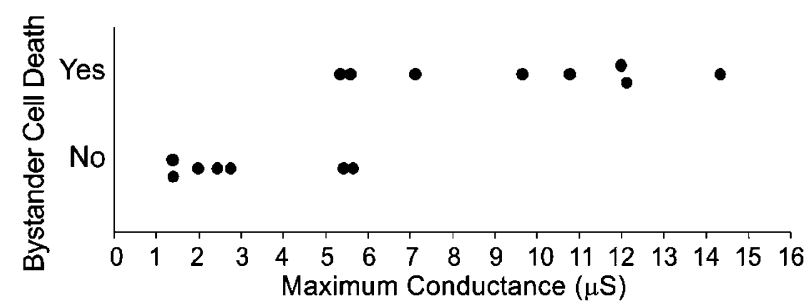

Figure 5 Maximum $G_{i}$ reached by each pair of cells from Figure 4(a-c) plotted against the outcome of the bystander cell (death or survival). The maximum conductance was highly correlated with bystander cell death, with a threshold where the outcome could go either way around $5-6 \mu \mathrm{S}$. Pairs with higher $G_{i}$ always underwent bystander cell death while pairs with lower $G_{j}$ never did cell death to determine the final conductance maximum (data not shown). As shown in Figure 6c, incubation in BAPTA-AM led to a dose-dependent rescue of bystander cells; $100 \mathrm{nM}$ rescued only one out of the six pairs that reached threshold, whereas $10 \mu \mathrm{M}$ rescued four out of five pairs. To determine whether $\mathrm{Ca}^{2+}$ release from internal stores plays a role in bystander cell death, we used Xestospongin $C$, an inhibitor of $\mathrm{IP}_{3}$ receptors. ${ }^{21}$ In all pairs treated with Xestospongin $\mathrm{C}$ that reached bystander cell death threshold $(n=4)$, the bystander cell was rescued, indicating that $\mathrm{IP}_{3}$ is an important mediator of this phenomenon (Figure $6 \mathrm{c}$ ). Xestospongin $\mathrm{C}$ slightly
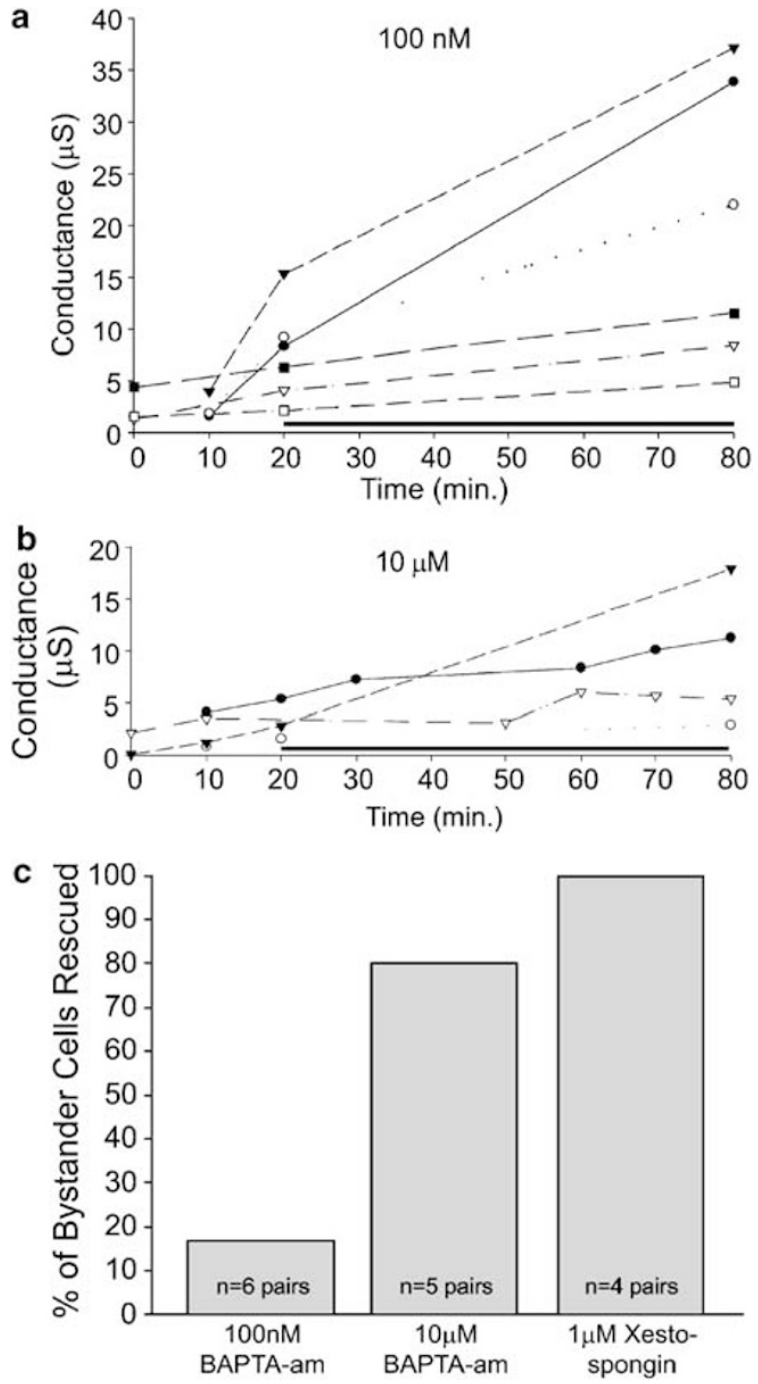

Figure 6 Pharmacological studies of the role of $\mathrm{Ca}^{2+}$ in bystander cell death. Pairs were incubated in BAPTA-AM or Xestospongin $\mathrm{C}$ for $1 \mathrm{~h}$ and washed out with modified Barth's solution. Both $100 \mathrm{nM}$ (a) and $10 \mu \mathrm{M}$ (b) BAPTA-AM increase $G_{i}$. Bar above $x$-axis indicates the time of BAPTA-AM incubation. (c) Inhibition of bystander cell death by BAPTA-AM and Xestospongin C. After washout with $\mathrm{MB}$, cells were injected with $\mathrm{cyC}$, and their coupling was followed if they were below the $6 \mu \mathrm{S}$ threshold. There is a dose-dependent response for BAPTA-AM. The number of pairs for each condition is indicated within the bar graph. Each experiment was replicated in multiple dishes. Bystander cells were considered 'rescued' if bystander cell death did not occur despite maximum conductance over $6 \mu \mathrm{S}$ 
decreased junctional conductance, but this effect was minimal (data not shown).

\section{Discussion}

This is the first study to measure directly the dynamic changes in $G_{j}$ between cells undergoing apoptosis and the cells to which they are electrically coupled. As we have shown, the introduction of cyC into one oocyte of a pair expressing the cell's endogenous connexin (Cx38) induces cell death in its neighbor, the bystander cell. The dying cells underwent swelling, and displayed an irregular mottled pattern of pigmentation and blistering at the animal pole (Figure 1a-d). Although apoptotic cells death is often associated with cell shrinkage owing to disruption of cytoplasmic structures, ion extrusion, DNA degradation, and nuclear fragmentation, it was shown that, in many of the cell lines tested, the early signs of cell death following microinjection of cyC consisted of plasma membrane budding, surface protrusions, and ultimately disruption of the cell membrane. ${ }^{18}$ The cell swelling seen in the present experiments appears to be even more pronounced, but it is important to recall that a requirement for the formation of gap junctional channels in paired oocytes is removal of both the follicular and vitelline membranes. Both membranes provide structural support for the cell, and serve as ionic barriers. In their absence, the gross intracellular changes associated with apoptosis probably induce an ionic imbalance that causes an influx of water and a resultant cell swelling.

Particularly noteworthy is the finding that for the uninjected cell to exhibit bystander cell death, it appears that, at least in oocytes, a threshold of conductance must be reached for the lethal agent to exert its effect (Figure 5). The lack of a conductance increase in apposed cells injected with antisense oligonucleotides to the endogenous connexin of Xenopus oocytes demonstrates that the cyC-induced conductance increase occurs only in the presence of $\mathrm{C} \times 38$, and therefore must require gap junctional coupling for transmission of apoptotic signals.

Also of interest is the observation that gap junctions remain open during cell death. As we have shown (Figure 2b), current recordings from electrically coupled cells before and after microinjection of cyC into one cell show that the gap junctional and total current (sum of junctional and nonjunctional currents) responses to hyperpolarizing voltage pulses increased in both cells over a $90 \mathrm{~min}$ time course, reaching a maximum at $\sim 70$ min postinjection. Only after the cells became uncoupled was there a precipitous drop in nonjunctional conductance. Moreover, the data clearly show that a threshold of conductance is necessary to kill the bystander cell; if the conductance did not reach this critical value $(\sim 6 \mu \mathrm{S})$ the coupled cell survived (Figure 5). The dose-response relationship between electrical coupling and bystander cell death is the clearest evidence obtained thus far that open gap junction channels mediate the spread of cell death. Although cell death occurred in every cell of an electrically coupled pair that was injected with cyC $(n=15)$, only about $50 \%$ of the uninjected cells displayed evidence of apoptosis. Some of this seeming inconsistency appears to result from the variability in junctional conductance displayed by the various cell pairs during the time frame over which the membrane integrity of the injected cell was lost. While it is evident that cyC served as the death-inducing vehicle in the injected cell, it cannot account for death of the uninjected cell, since the molecular mass of cyC $(12.3 \mathrm{kDa})$ is too large to pass through the gap junctional pores. Although the death signal was passed intercellularly, the nature of the toxic agent was not identified unequivocally. Nevertheless, the fact that agents which bind $\mathrm{Ca}^{2+}$ (BAPTA) or suppress its release from $\mathrm{IP}_{3}$-sensitive intracellular stores (Xestospongin $\mathrm{C}$ ) serve to inhibit cell death (Figure 6) suggests that transfer of $\mathrm{Ca}^{2+}$ or $\mathrm{IP}_{3}$, both of which are gap junction permeant, ${ }^{22}$ may be a contributing factor.

Previous studies have shown that gap junction mediated cell death occurs in many cell types in response to different death-inducing stimuli. ${ }^{5,8,11,16,23}$ Dye-transfer between cells has been reported during cell death ${ }^{17}$ and has been shown to decrease during ischemia, ${ }^{16,24}$ but the dynamics of gap junctional coupling during cell death have not been studied previously.

Cotrina et al. ${ }^{24}$ examined currents from single astrocytes in cortical slices and suggested that the difference in currents between cells on the surface of the slice, which were not dyecoupled, and those deeper in the slice that were dye-coupled could be attributed to gap junctional currents. This indirect measure of gap junctional coupling was shown to decrease during ischemia, but some current was retained. These results are in contrast with the studies presented here which show an increase in both $G_{j}$ between cells and $G_{n j}$ in dying cells. However, it is possible that the cells they were recording from were not undergoing cell death or directly coupled to dying cells as they did not establish cell death in the individual cells they were recording from. Rather, the cells may be responding to changes in their extracellular environment, which may result from the necrosis of neighboring but uncoupled cells. Alternatively, the type of death stimulus or cell type-specific responses may account for these disparate results.

There is considerable evidence that intercellular communication through gap junction channels may not only be detrimental but may also be beneficial to survival of surrounding cells (the Good Samaritan effect). ${ }^{25}$ Studies on heterozygous $\mathrm{Cx} 43^{+/-}$mice have revealed that the reduction of expression of this astrocytic gap junction protein by $50 \%$ results in larger infarct volume of lesions but smaller astrogliosis 4 days after cerebral artery occlusion. ${ }^{26}$ Moreover, myocardial infarcts are smaller in $\mathrm{C} \times 43^{+/-}$mice 8 days and 10 weeks after coronary occlusion ${ }^{27}$ and $\mathrm{Cx} 43$ null osteoblasts showed higher camptothecin-induced apoptosis. ${ }^{28}$ Mild, nonlethal oxidative and thermal stress have been shown to increase both dye coupling and the recycling of connexin proteins to the membrane, ${ }^{29}$ suggesting that coupling may increase in disease conditions in which cells are under oxidative or metabolic stress. Increasing coupling may allow metabolic cooperation or diffusion of toxic metabolites across the volume of multiple cells, with the possible effect of rescuing the initial cell from dying, but also with the potential to kill the coupled cells.

In sum, the present study is the first to provide a quantitative assessment of the relation between junctional conductance and gap junction-mediated cell death. The results show that 
gap junctions remain open during cell death, that gap junctiondependent bystander cell death occurs, and that intercellular transmission or mobilization of $\mathrm{Ca}^{2+}$ is probably a significant factor in the death of bystander cells.

\section{Materials and Methods}

\section{Oocyte isolation and preparation}

All experimental procedures adhered to the ARVO Statement for the Use of Animals in Ophthalmic and Vision Research, and conformed to the guidelines for the Care and Use of Laboratory Animals established by the Animal Care Committee of the UIC College of Medicine. Methods for oocyte preparation and electrophysiological recording have been described previously. ${ }^{30}$ Briefly, oocytes were removed from gravid $X$. laevis females (Xenopus One, Dexter, MI, USA), and defolliculated by incubation in a calcium-free modified Barth's (MB) solution containing collagenase $(2.5 \mathrm{mg} / \mathrm{ml})$ for $2 \mathrm{~h}$ under constant agitation. Stage $\mathrm{V}-\mathrm{VI}$ oocytes were selected, and stored at $15^{\circ} \mathrm{C}$ in $\mathrm{MB}$ containing (in $\mathrm{mM}$ ) $\mathrm{NaCl}$ $88, \mathrm{KCl} 1, \mathrm{NaHCO}_{3} 2.4, \mathrm{~N}$-2-hydroxyethylpiperazine- $N$-2-ethanesulfonic acid (HEPES) $15, \mathrm{Ca}\left(\mathrm{NO}_{3}\right)_{2} \quad 0.33, \mathrm{CaCl}_{2} 0.40$, glucose 5 , and $\mathrm{MgSO}_{4}$ $0.82 ; 10 \mathrm{mg} / \mathrm{l}$ gentamycin was added, and the solution titrated with $\mathrm{NaOH}$ to $\mathrm{pH}$ 7.6. All chemicals were purchased from Sigma, St Louis, MO, USA, with the exception of gentamycin, which was from Gibco/BRL-Invitrogen, Carlsbad, CA, USA.

To suppress the activity of gap junctional channels formed by the endogenous connexin (Cx38) of Xenopus oocytes, ${ }^{31}$ the oocytes were injected with $46 \mathrm{nl}$ of an aqueous solution containing $10 \mathrm{ng}$ of an antisense oligomer to nucleotides 128-151 of the coding region of Cx38 (5'CTG ACT GCT CGT CTG TCC ACA CAG-3') using a Nanoject II oocyte injector (Drummond, Broomall, PA, USA). The oocytes were stripped of their vitelline membranes in a hypertonic solution, ${ }^{32}$ placed on the concave surface of cylindrical Teflon channels that were adherent to the base of a $35 \mathrm{~mm}$ culture dish, and paired at their vegetal poles for $24-48 \mathrm{~h}$ before electrophysiological study.

\section{Cytochrome $c$-induced cell death}

The use of cyC to induce cell death is now a relatively common procedure, ${ }^{4-6,19,20,33}$ and provides a convenient means to circumvent the need for various extracellular signaling molecules that activate membrane receptors of the death domain family. Cytoplasmic cyC is an important component of the apoptotic cascade, and its release from mitochondria precedes exposure of phosphatidylserine, the loss of plasma membrane integrity, and other indices of apoptosis. ${ }^{34,35}$ More importantly, the intracellular machinery of apoptosis is bypassed when cyC is introduced intracellularly, whereupon it forms a complex (the so-called apoptosome) with Apaf- 1 , procaspase- 9 and dATP. The resultant cleavage and activation of caspase 9 leads, in turn, to the activation of downstream executioner caspases (e.g., caspase 3) that cause DNA damage, disruption of cytoskeletal proteins and the stereotypical changes that characterize apoptotic cell death. ${ }^{33}$ In the present study, every cell injected with cyC $(n=>50)$ initially showed signs of cell death within a short period ( $<30 \mathrm{~min}$ ), while $1-2 \mathrm{~h}$ were required for complete degeneration following the injection.

\section{Electrical recordings}

A dual, two electrode voltage clamp paradigm was used to record junctional currents in oocyte pairs. ${ }^{36,37}$ Microelectrodes (thin-wall, $1.2 \mathrm{~mm}$ outside diameter, WPI, Sarasota, FL, USA) were drawn on a vertical puller (Narashige, Tokyo, Japan) to a resistance of $0.7-1.5 \mathrm{M} \Omega$ when filled with $3 \mathrm{M} \mathrm{KCl}$, and connected to the input stages of two GeneClamp 500B amplifiers (Axon Instruments, Foster City, CA, USA). The voltage clamp was under computer control through a Digidata 1322A interface (Axon), and voltage protocols were provided by a Master Eight stimulator (AMPI, Jerusalem, Israel) through programs generated in pClamp 8 software (Axon). To quantitate junctional conductance $\left(G_{j}\right)$, the two cells were clamped initially at $-40 \mathrm{mV}$, close to their resting potential, and transjunctional current measurements were obtained in response to $20 \mathrm{mV}$ hyperpolarizing pulses of $1 \mathrm{~s}$ duration applied alternately to the two cells with a delay of $10 \mathrm{~s}$; the paired pulses were delivered at $30 \mathrm{~s}$ intervals. Under these conditions, the current supplied by the clamp to the follower cell provides a measure of the junctional current, and the junctional conductance is then readily calculated by dividing the junctional current by the transjunctional potential. Junctional currents were measured two or three times to obtain baseline data before one oocyte of the pair was injected with $173 \mathrm{ng}$ of cyC (Sigma) in a volume of $46 \mathrm{nl}$ to induce cell death. Oocyte volume estimates range from $\sim 500 \mathrm{nl}^{38}$ to $1 \mu \mathrm{l}^{20}$ which would put the final intracellular concentration of cyC $(M W=12,327)$ in the range of $14-28 \mu \mathrm{M}$. The voltage step protocols were run for approximately two minutes at $10 \mathrm{~min}$ intervals until the cells could no longer be voltage clamped. Gap junctional currents were measured from at least four individual episodes at each time point after the current had reached steady state, and the junctional conductance was calculated from the mean current of the four episodes. All Gis shown were calculated from current measurements in the noninjected cell during voltage pulses delivered to the cyC-injected cell. $G_{i}$, nonjunctional conductance, and input resistance were calculated in Microsoft Excel, and graphs were prepared in Sigma Plot 2000 or Origin 6.0 (Microcal Inc. Northhampton, MA, USA).

\section{Pharmacological studies}

A series of control experiments were conducted initially to determine the effect of the calcium chelator BAPTA-AM on the induction of cell death by cyC. When single (unpaired) cells were injected with cyC following a $1 \mathrm{~h}$ incubation in the presence or absence of $10 \mu \mathrm{M}$ BAPTA-AM $(n=5$ per condition), cell death occurred in all of the injected cells, and the loss of membrane potential proceeded along the same time course; vehicleinjected cells did not die in either condition. The results of these preliminary studies indicate that chelation of $\mathrm{Ca}^{2+}$ does not induce or interfere with cyC -induced cell death, nor does it render cells more susceptible to the insult of injection.

With cell pairs, baseline $G_{j}$ was measured prior to drug treatment to ensure electrical coupling, and the paired cells were loaded for $1 \mathrm{~h}$ with BAPTA-AM $(100 \mathrm{nM}$ or $10 \mu \mathrm{M})$ in a solution containing $90 \mathrm{mM} \mathrm{KCl}, 1 \mathrm{mM}$ EGTA, and $5 \mathrm{mM}$ HEPES, adjusted to $\mathrm{pH} 7.5$ with $\mathrm{KOH}$. After washout with $\mathrm{MB}$, gap junctional currents were again measured, and one cell was injected with cyC to induce cell death. In related experiments, cells were incubated for $30 \mathrm{~min}$ in $1 \mu \mathrm{M}$ Xestospongin $\mathrm{C}$ (Sigma) and junctional currents were measured prior to and following cyC injection.

\section{Acknowledgements}

This work was supported by the NIH Grants HL07675 (KC); EY-06516, EY-01792 and EY-14557 (HR); MH-65495 (DCS), a Grass Foundation Fellowship (KC), an unrestricted award to the UIC Department of Ophthalmology and Visual Sciences from Research to Prevent Blindness Inc., a Senior Research Investigator Award from RPB (HR), and an Award 
of Merit from the Alcon Research Institute (HR). We thank Dr. Julie Schnapf for helpful discussions.

\section{References}

1. Oppenheim R (1991) Cell death during development of the nervous system. Annu. Rev. Neurosci. 14: 453-501.

2. Stone J, Maslim J, Valter-Kosci K, Mervin K, Bowers F, Chu Y, Barnett N, Provis J, Lewis G, Fisher SK, Bisti S, Gargini C, Cervetto L, Merin S, Peer J (1999) Mechanisms of photoreceptor death and survival in mammalian retina. Prog. Retin. Eye Res. 18 (6): 689-735.

3. Linden R (2000) The anti-death league: associative control of apoptosis in developing retinal tissue. Brain Res. Brain Res. Rev. 32: 146-158.

4. Cusato K, Bosco A, Rozental R, Guimarães $C A$, Reese BE, Linden R, Spray DC (2003) Gap junctions mediate bystander cell death in developing retina. J. Neurosci. 23: 6413-6422.

5. Cusato K, Zakevicius J, Ripps H (2003) An experimental approach to the study of gap-junction-mediated cell death. Biol. Bull. 205: 197-199.

6. Udawatte C, Ripps $\mathrm{H}(2005)$ The spread of apoptosis through gap-junctional channels in BHK cells transfected with Cx32. Apoptosis 10: 1019-1029.

7. Freeman SM, Abboud CN, Whartenby KA, Packman CH, Koeplin DS, Moolten FL, Abraham GN (1993) The 'bystander effect': tumor regression when a fraction of the tumor mass is genetically modified. Cancer Res. 53: 5274-5283.

8. Mesnil M, Piccoli C, Tiraby G, Willecke K, Yamasaki H (1996) Bystander killing of cancer cells by herpes simplex virus thymidine kinase gene is mediated by connexins. Proc. Nat. Acad. Sci. USA 93: 1831-1835.

9. Mesnil M, Yamasaki H (2000) Bystander effect in herpes simplex virusthymidine kinase/ganciclovir cancer gene therapy: role of gap-junctional intercellular communication. Cancer Res. 60: 3989-3999.

10. Tanaka T, Yamasaki H, Mesnil M (2001) Stimulation of intercellular communication of poor-communicating cells by gap-junction-competent cells enhances the HSV-TK/GCV bystander effect in vitro. Int. J. Cancer 91: 538-542.

11. Elshami AA, Saavedra A, Zhang H, Kucharczuk JC, Spray DC, Fishman GI, Amin KM, Kaiser LR, Albelda SM (1996) Gap junctions play a role in the 'bystander effect' of the herpes simplex virus thymidine kinase/gancyclovir system in vitro. Gene Therapy 3: 85-92.

12. Estin D, Li M, Spray DC, Wu JK (1999) Connexins are expressed in primary brain tumors and enhance the bystander effect in gene therapy. Neurosurgery 44: 361-368; discussion 368-369.

13. Simpson I, Rose B, Loewenstein WR (1977) Size limits of molecules permeating the junctional membrane channels. Science 195: 18596-18603.

14. Bennett MV, Barrio LC, Bargiello TA, Spray DC, Hertzberg E, Saez JC (1991) Gap junctions: new tools, new answers, new questions. Neuron 6: 305-320.

15. Sosinsky GE, Perkins GA (2000) Electron crystallographic methods for investigating gap junction structure. Methods 20: 140-155.

16. Lin JH, Weigel H, Cotrina ML, Liu S, Bueno E, Hansen AJ, Hansen TW, Goldman S, Nedergaard M (1998) Gap-junction-mediated propagation and amplification of cell injury. Nat. Neurosci. 1: 494-500.

17. Wilson MR, Close TW, Trosko JE (2000) Cell population dynamics (apoptosis, mitosis, and cell-cell communication) during disruption of homeostasis. Exp. Cell Res. 254: 257-268.

18. Brustugun OT, Fladmark KE, Doskeland SO, Orrenius S, Zhivotovsky B (1998) Apoptosis induced by microinjection of cytochrome $c$ is caspase-dependent and is inhibited by Bcl-2. Cell Death Differ. 5: 660-668.
19. Zhivotovsky B, Orrenius S, Brustugun OT, Døskeland SO (1998) Injected cytochrome $c$ induces apoptosis. Nature (London) 391: 449-450.

20. Bhuyan AK, Varshney A, Mathew MK (2001) Resting membrane potential as a marker of apoptosis: studies on Xenopus oocytes microinjected with cytochrome c. Cell Death Differ. 8: 63-69.

21. Gafni J, Munsch JA, Lam TH, Catlin MC, Costa LG, Molinski TF, Pessah IN (1997) Xestospongins: potent membrane permeable blockers of the inositol 1,4,5-trisphosphate receptor. Neuron 19: 723-733.

22. Saez JC, Connor JA, Spray DC, Bennett MV (1989) Hepatocyte gap junctions are permeable to the second messenger, inositol 1,4,5-trisphosphate, and to calcium ions. Proc. Natl. Acad. Sci. USA 86: 2708-2712.

23. Vrionis FD, Wu JK, Qi P, Waltzman M, Cherington V, Spray DC (1997) The bystander effect exerted by tumor cells expressing the herpes simplex virus thymidine kinase (HSVtk) gene is dependent on connexin expression and cell communication via gap junctions. Gene Therapy 4: 577-585.

24. Cotrina ML, Kang J, Lin JH, Bueno E, Hansen TW, He L, Liu Y, Nedergaard M (1998) Astrocytic gap junctions remain open during ischemic conditions. J. Neurosci. 18: 2520-2537.

25. Andrade-Rozental AF, Rozental R, Hopperstad MG, Wu JK, Vrionis FD, Spray DC (2000) Gap junctions: the 'kiss of death' and the 'kiss of life'. Brain Res. 32 308-315.

26. Nakase T, Fushiki S, Naus CC. (2003) Astrocytic gap junctions composed of connexin 43 reduce apoptotic neuronal damage in cerebral ischemia. Stroke 34: 1987-1993.

27. Kanno S, Kovacs A, Yamada KA, Saffitz JE (2003) Connexin43 as a determinant of myocardial infarct size following coronary occlusion in mice. J. Am. Coll. Cardiol. 41: 681-686.

28. Furlan F, Lecanda F, Screen J, Civitelli R (2001) Proliferation, differentiation and apoptosis in connexin43-null osteoblasts. Cell Commun. Adhes. 8: 367-371.

29. VanSlyke JK, Musil LS (2002) Dislocation and degradation from the ER are regulated by cytosolic stress. J. Cell Biol. 157: 381-394.

30. Ripps H, Qian H, Zakevicius J (2002) Pharmacological enhancement of hemi-gap-junctional currents in Xenopus oocytes. J. Neurosci. Meth. 121: 81-92.

31. Ebihara L, Beyer EC, Swenson KI, Paul DL, Goodenough DA (1989) Cloning and expression of a Xenopus embryonic gap junction protein. Science 243: 1194-1195

32. Methfessel C, Witzemann V, Takahashi T, Mishina M, Numa S, Sakmann B (1986) Patch clamp measurements on Xenopus laevis oocytes: currents through endogenous channels and implanted acetylcholine receptor sodium channels. Pflugers Arch. 407: 577-588.

33. Li F, Srinivasan A, Wang Y, Armstrong RC, Tomaselli KJ, Fritz LC (1997) Cellspecific induction of apoptosis by microinjection of cytochrome $c$. J. Biol. Chem 272: 30299-30305.

34. Liu X, Kim CN, Yang J, Jemmerson R, Wang X (1996) Induction of apoptotic program in cell free extracts: requirement for dATP and cytochrome $c$. Cell 86 : $147-157$.

35. Jiang X, Wang X (2004) Cytochrome c-mediated apoptosis. Ann. Rev. Biochem. 73: 87-106.

36. Dahl G, Azarnia R, Werner R (1981) Induction of cell-cell channel formation by mRNA. Nature (London) 289: 683-685.

37. Spray DC, Harris AL, Bennett MVL (1981) Gap junctional conductance is a simple and sensitive function of intracellular $\mathrm{pH}$. Science 211: 712-714.

38. Zhang Y, Hamill OP (2000) Calcium-, voltage- and osmotic stress-sensitive currents in Xenopus oocytes and their relationship to single mechanically gated channels. J. Physiol. 523 (Part 1): 83-99. 